\title{
LOW LEVEL LASER THERAPY (LLLT) FOR ELDERLY RESIDENTS IN FACILITIES OF HEALTH CARE SERVICES
}

\author{
Yasuo Ito ${ }^{1}$, Ikuko Ohkuni ${ }^{2}$, Nobuyuki Ushigome ${ }^{2}$, Takashi Harada ${ }^{2}$, \\ Hiroshi Tsuruoka ${ }^{2}$, Go Endo ${ }^{3}$, Kane Suzuki ${ }^{3}$ \\ 1: Yamakita Clinic, Kochi, Japan \\ 2: Department of Physical Medicine and Rehabilitation, Toho University, Tokyo, Japan \\ 3: Institute of Sosei Social Welfare Work, Fukushima, Japan
}

\begin{abstract}
The present study examines the usefulness of low level laser therapy (LLLT) given to residents in nursing homes for the elderly. In many cases the elderly have complications brought about by disuse. The disuse syndrome is a general term describing unhealthy symptoms caused by a considerable decrease in activity. The main symptoms include chronic pain, decubitus ulcers, cognitive disorders, deterioration in respiratory and circulatory functions, pneumonia, constipation, urinary tract infections, muscular dystrophy, bone atrophy, and so on. From these symptoms, we confirmed LLLT is effective for decubitus ulcers and improves the patients' quality of life (QOL). However, only $10 \%$ of the patients, 2 of the 20 patients we treated, could return to their homes.
\end{abstract}

Key Words: Nursing home for the elderly; Elderly Patients; Low Level Laser Therapy; Disuse Syndrome

\section{Introduction}

About twenty years ago, the era of the traditional elderly lifestyle, whereby they could spend their remaining years comfortably at home with, band being looked after by, their family, started to change. Due to swift changes in the social structure caused by a rapidly aging Japanese society and the entry of all family members into the workforce, the elderly have lost the traditional lifestyle when they were able to stay with their families. As a matter of fact, in many cases it has become unavoidable for them to either live alone, or move into facilities of health care services for the elderly, commonly referred to as nursing homes. We report herein on the results of our therapeutic study of

Addressee for Correspondence:

Takashi Harada

Department of Rehabilitation Medicine

Toho University School of Medicine

6-11-1 Omori-nishi, Ohta-ku, Tokyo, 143-8541, Japan

TEL: +81-3-3762-4151

FAX: +81-3-3768-6117

E-mail: haradata@med.toho-u.ac.jp

(C2010 JMLL, Tokyo, Japan low level laser therapy (LLLT) applied to twenty subjects out of 100 residents at a nursing home for the elderly.

\section{Subjects and Methods}

\section{Subjects}

Twenty subjects suffering from musculoskeletal pain, sequelae following cerebral vascular accidents (CVAs), or decubitus ulcers were selected from among 100 residents of a nursing home for the elderly in Fukushima prefecture. Their ages ranged from seventy-two to ninety years, with an average of eighty-four years. The patients had complications from several diseases, although their conditions were somewhat eased for at least some period with medications, and nursing care. The clinical features of the selected patients are shown in Table 1.

Manuscript received: March 1st, 2010

Accepted for publication: April 26th, 2010 
Table 1. Patient Outlines

\begin{tabular}{|c|c|c|c|c|c|}
\hline Case & Name & Age & Sex & Symptoms & Complications \\
\hline 1 & K.S. & 87 & $\mathrm{~F}$ & knee joint pain & CVA* $^{*}$ \\
\hline 2 & M.K. & 81 & $\mathrm{~F}$ & shoulder and hand pain & CVA \\
\hline 3 & S.S. & 90 & M & decubitus & spodylosis \\
\hline 4 & T.S. & 75 & $\mathrm{~F}$ & decubitus & CVA \\
\hline 5 & T.K. & 90 & $\mathrm{~F}$ & knee joint pain & CVA \\
\hline 6 & T.S. & 90 & $\mathrm{~F}$ & knee joint pain & spodylosis \\
\hline 7 & K.T & 90 & $\mathrm{~F}$ & knee joint pain & cognitive disorder \\
\hline 8 & K.K. & 82 & M & decubitus & cognitive disorder \\
\hline 9 & Y.Y. & 72 & M & knee joint pain & Parkinson's \\
\hline 10 & Y.S. & 80 & $\mathrm{~F}$ & knee joint pain & CVA \\
\hline 11 & Y.M. & 88 & $\mathrm{~F}$ & decubitus & cognitive disorder \\
\hline 12 & T.S. & 67 & $\mathrm{~F}$ & decubitus & anterior spinal artery syndrome \\
\hline 13 & T.K & 82 & $\mathrm{~F}$ & knee joint pain & CVA \\
\hline 14 & R.S. & 90 & $\mathrm{~F}$ & decubitus & CVA \\
\hline 15 & M.S. & 81 & $\mathrm{~F}$ & knee joint pain & cervical myelopathy \\
\hline 16 & S.M. & 76 & $\mathrm{~F}$ & knee joint pain & CVA \\
\hline 17 & T.I. & 90 & $\mathrm{~F}$ & decubitus & CVA \\
\hline 18 & K.S. & 90 & $\mathrm{~F}$ & knee joint pain & CVA \\
\hline 19 & K.S. & 90 & $\mathrm{~F}$ & back pain & osteoporosis \\
\hline 20 & Y.S. & 90 & $\mathrm{~F}$ & knee joint pain & CVA \\
\hline
\end{tabular}

${ }^{*}$ CVA: Cerebral vascular accident

Table 2. Low Level Laser Therapy Device Specifications

\begin{tabular}{cl}
\hline Laser Element & Semiconductor Laser Diode (GaAlAs: Gallium Aluminum Arsenide) \\
\hline Model & MDL-2001 model \\
Manufacturer & Matsushita Electric Corporation, Tokyo, Japan \\
\hline Wavelength & $830 \pm 15 \mathrm{~nm}$ \\
Output & $1000 \mathrm{~mW} \pm 20 \%$ \\
Mode & Continuous wave mode \\
\hline Spot size & Diameter: $14 \mathrm{~mm}$, Area: $1.5 \mathrm{~cm}^{2}$ \\
\hline Irradiation time & $30 \mathrm{sec}$ \\
\hline Energy density & $20.1 \mathrm{~J} / \mathrm{cm}^{2}$ \\
\hline Power Supply & $100 \mathrm{VAC}, 50-60 \mathrm{~Hz}$ \\
\hline
\end{tabular}

\section{Methods}

We used a diode-based low level laser therapy device with an output of $1000 \mathrm{~mW}$, and the full specifications are as shown in Table 2.

\section{Targets for laser therapy}

Low level laser therapy (LLLT) was applied to the tender points and the decubitus ulcers four times for fifteen seconds per visit. Patients had this treatment twice a week for three months. We used contact mode with positive pressure.

\section{Evaluation}

Since we often could not understand what the elderly patients were trying to say, we were not able to evaluate the pre- and postirradiation pain score accurately with the usual visual analogue scale (VAS). Therefore, statistical analysis was not applied to the pain data. We evaluated the patient's subjective satisfaction level on 


\section{ORIGINAL ARTICLES}

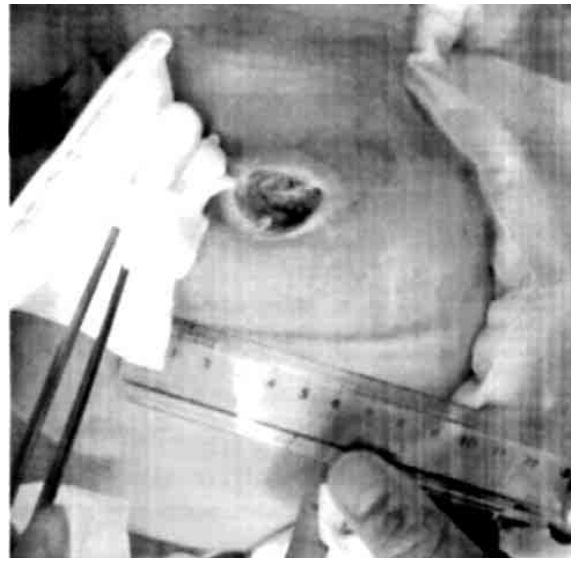

first visit

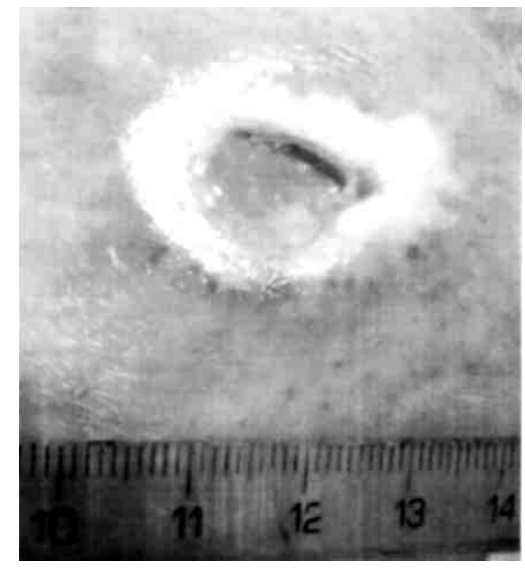

after one month

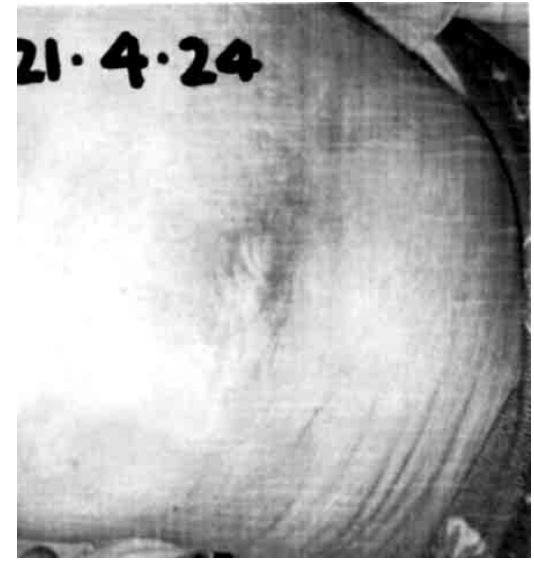

after two months

Figure 1: CVA patient with decubitus (Case no 14)

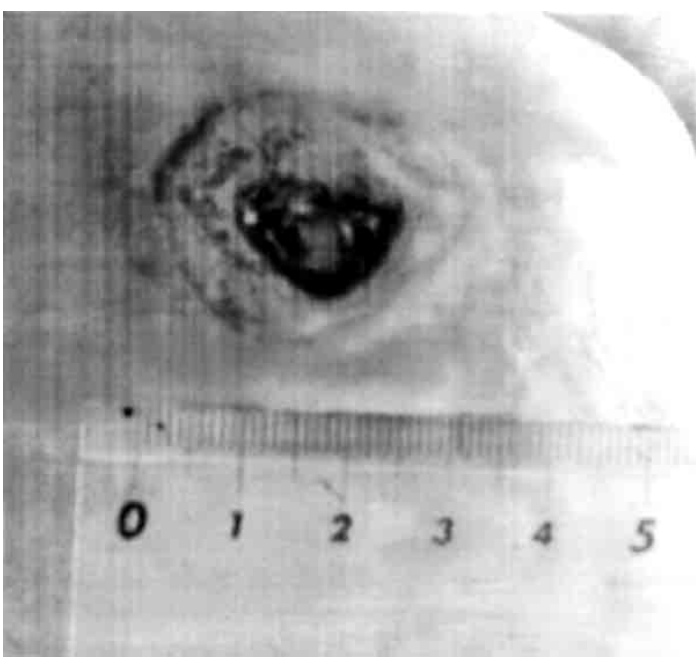

first visit

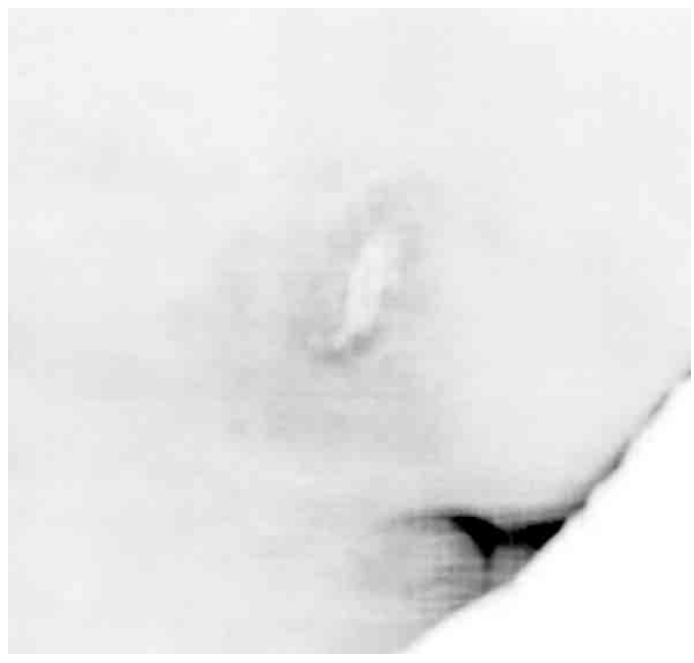

after three months

Figure 2: Patient with spinal cord injury due to anterior spinal artery syndrome (Case no 12)

4-item Excellent, Good, Little or no change, and exacerbation or other undesirable side effects. Range of motion (ROM) was measured by the same person before and after the irradiation. After having had the purpose and procedures of the trial explained to them and/or their families, all patients, and where applicable their families, gave written informed consent to participate in the study.

\section{Results}

Nineteen cases out of 20 evaluated their pain attenua- tion as "Excellent." One case had numbness caused by a cerebral vascular accident (CVA), and was evaluated as "No change." There was no change in range of motion (ROM) in any of the patients after irradiation.

The treatment processes for two cases with intractable decubitus ulcers are shown in Figure 1 and 2. In Case 1, a 90 year-old female (Figure 1), healing occurred after two months. The decubitus ulcer in Case 2, a 67 year-old female, took three months to heal. Only ten percent out of 20 cases could return to their homes. 


\section{Discussion}

In 2000, a system of nursing care insurance was enforced in Japan to allow the elderly to be more independent with their activities of daily living in nursing homes. ${ }^{1)}$ One goal was for them to eventually resume living at home. However, in reality, it is difficult for residents in nursing homes for the elderly to resume their places at home or in society because their disuse syndrome gradually worsens due to aging. ${ }^{2-5)}$ The percentage of residents who eventually were able to return home in the facility in the present study was only ten percent, which turned out to be around the same percentage as the average for facilities throughout Japan.

Disuse syndrome ${ }^{6-9)}$ is a general term describing the psychosomatic disease state that causes an unhealthy condition over the whole body due to a significant decline in activity. The main symptoms of disuse syndrome are cognitive disorders, sleeping disorders, aspiration pneumonia, constipation, urinary tract infections, muscle atrophy, bone atrophy, joint contracture, skin atrophy, decubitus ulcers, chronic pain, and so on. We could not find any reports about LLLT for elderly resi-

\section{References}

1: White Paper of Ministry of Health, Labour and Welfare(1999). Law of Care Insurance of the Elderly in 1999.

2: Nagata K (2009): Risk of Clinical Medicine in the Facility of Health Care Services or the Elderly. Care and Welfare, 74: 51-64.

3: Rowe JW, Kahn RL (1998): Successful Aging. New York, Dell Pub p.39.

4: Haga H (2004: Rehabilitation on Care Management and Facility of Health Care Services for the Elderly. Science Cooperating with Rehabilitation 5: 2-14.

5: Asayama K (2008): Overview-an abusive use of the disuse syndrome. $\mathrm{J}$ of Clinical Rehabilitation, 17 : 118-122.

6: Sanada H (1998): Prevention of Pressure Ulcer. Report of Division of Health Care Services for the Elderly, Welfare and Health Administration. Shorinsha, Tokyo. pp 12-14.

7: Suyama S (2008): Medical Characteristics and Care of the Patients with Pressure Ulcers 10:573-585. dents in nursing homes in the literature, so to the best of our knowledge, this is the first study to report on this subject.

Reported physical reactions to LLLT are alleviation of pain, 10,11) blood circulation improvement, ${ }^{12)}$ autonomic nerve adjustment, promotion of collagen fiber building, immune system improvement, regeneration of peripheral nerves, ${ }^{13)}$ and so on. In all 100 cases, we saw some manifestations of disuse syndrome. In this investigation, we explained and received consent from 20 cases. For chronic pain and decubitus ulcers, LLLT at the parameters used in the present study was effective for improving the quality of life of the elderly trial subjects. The elderly patients' high satisfaction resulted in greater motivation to live. In addition, their families' level of satisfaction was also high. Consequently, the patients' QOL improved. We confirmed LLLT treatment to be especially effective for blood circulation disorders resulting in decubitus ulcers, and LLLT helped to promote wound healing in the previously intractable ulcers through a photomediated return to normal collagen fiber synthesis. Concerning no change in ROM, we considered that their patients had already serious joint contractures.

8: Tsuji I (2006): Disability prevention and disuse syndrome. Sogo Rehabilitation, 34: 649-653.

9: Terai S, Miyamoto H (2007): Present status of patients in a rehabilitation unit at a geriatric hospital. Nippon Ronen Igakkai Zasshi, 44: 476-482.

10: Klein RG (1990): Low energy laser treatment and exercise for chronic low back pain. Ach Phys Med Rehabil, 71: 34-47.

11: Shiroto C, Yodono C, Nakaji S (1998): Pain attenuation with diode laser therapy. Laser Therapy, 10: 33-40.

12: Maegawa Y, Itoh T, Hosokawa T (2000): Effects of near infrared low level laser irradiation on microcirculation. Laser in Surgery and Medicine 27:427437.

13: Anders JJ, Borke RC, Woolery S (1991): Low energy laser irradiation alters immuno-fluorescence of choline acetyltransferase (CHAT) in regenerating rat facial neurons. Neurosci, 17: 1483. 